\title{
Localization of interacting Fermi gases in quasiperiodic potentials
}

\author{
Sebastiano Pilati ${ }^{1,2}$ and Vipin Kerala Varma ${ }^{1}$ \\ ${ }^{1}$ The Abdus Salam International Centre for Theoretical Physics, 34151 Trieste, Italy \\ ${ }^{2}$ Scuola Normale Superiore, 56126 Pisa, Italy \\ (Received 24 October 2016; published 17 January 2017)
}

\begin{abstract}
We investigate the zero-temperature metal-insulator transition in a one-dimensional two-component Fermi gas in the presence of a quasiperiodic potential resulting from the superposition of two optical lattices of equal intensity but incommensurate periods. A mobility edge separating (low-energy) Anderson localized and (high-energy) extended single-particle states appears in this continuous-space model beyond a critical intensity of the quasiperiodic potential. To discern the metallic phase from the insulating phase in the interacting manyfermion system, we employ unbiased quantum Monte Carlo (QMC) simulations combined with the many-particle localization length familiar from the modern theory of the insulating state. In the noninteracting limit, the critical optical-lattice intensity for the metal-insulator transition predicted by the QMC simulations coincides with the Anderson localization transition of the single-particle eigenstates. We show that weak repulsive interactions induce a shift of this critical point towards larger intensities, meaning that repulsion favors metallic behavior. This shift appears to be linear in the interaction parameter, suggesting that even infinitesimal interactions can affect the position of the critical point.
\end{abstract}

DOI: 10.1103/PhysRevA.95.013613

To what extent, if at all, do Anderson insulators persist in the presence of interactions? This has been an outstanding problem since 1958 when noninteracting quantum systems were theoretically shown by Anderson to harbor no transport of conserved quantities for sufficiently strong disorder [1,2].

In cold-atom settings, among others, experimenters have observed the Anderson transition of noninteracting particles either in the random disorder created by using speckle patterns (which are characterized by finite-range spatial correlations) or in the one-dimensional (deterministic) quasidisorder created by using incommensurate bichromatic lattices [3-7]. Theoretical predictions about the critical point of the Anderson transition based on models that take into account the details of these cold-atom experiments have been recently reported [8-11], enabling quantitative comparison with experimental measurements [12].

Cold-atom experiments have emerged as the ideal playground to also explore the effects due to interactions in disordered many-body systems $[13,14]$. Experiments to understand the transport and localization phenomena in disordered interacting atomic gases continue to be performed [15-22]. Theoretically, a decade ago, Basko and collaborators showed by using diagrammatic techniques that the Anderson insulator can survive in the presence of interactions [23], even at finite temperatures [24] (see Ref. [25] for a numerical study on spin chains). For continuous-space disordered bosons this finite-temperature localization [26] connects, in the zerotemperature limit, to the superfluid to Bose glass transition [27]. The concomitant zero-temperature localization transition for continuous-space weakly interacting quasidisordered fermions is the subject of our study.

In this paper, we investigate the zero-temperature metalinsulator transition in a one-dimensional two-component Fermi gas with contact repulsive interactions. We consider a realistic continuous-space model for a cold-atom setup where an atomic Fermi gas is subjected to the quasiperiodic potential created by the superposition of two periodic optical lattices with the same intensity but with incommensurate periods.
Similarly to the related (discrete-lattice) Aubry-André model [28], which would describe this physical system if one of the two optical lattices was very deep and the other extremely weak, the single-particle spectrum of this (continuous-space) model displays an Anderson transition where (part of) the eigenstates become spatially localized; however, in contrast to the Aubry-Andre model, here there is a mobility edge which separates the localized state with energies below the mobility edge from the extended ergodic states above it $[29,30]$.

To discern the metallic phase from the insulating phase we adopt the concepts familiar from the modern theory of the insulating state [31]; in particular, the expectation value of the many-body phase operator [32]. This approach allows one to distinguish metals from insulators by inspecting ground-state properties, i.e., without direct computation of low-lying exited states or dynamical properties. In the interacting case, we compute this quantity via unbiased quantum Monte Carlo simulations based on the diffusion Monte Carlo algorithm, which is suitable for simulating large-scale realistic models, paving the way for quantitative comparison with experiments in interacting atomic gases. Our main goal is to inspect the effects of weak interactions on the critical point of the Anderson transition, i.e., whether it drifts towards stronger or weaker intensities of the quasiperiodic potential, or instead if it is insensitive to interactions below a certain threshold.

The one-dimensional atomic Fermi gas we consider is described by the following continuous-space Hamiltonian:

$$
\hat{H}=\sum_{i=1}^{N}\left(-\frac{\hbar^{2}}{2 m} \frac{d^{2}}{d x_{i}^{2}}+v\left(x_{i}\right)\right)+\sum_{i_{\uparrow}, i_{\downarrow}} g \delta\left(x_{i_{\uparrow}}-x_{i_{\downarrow}}\right),
$$

where $\hbar$ is the reduced Planck constant, $m$ is the atomic mass, $N_{\uparrow}$ and $N_{\downarrow}$ are the numbers of atoms of the two components-hereafter referred to as spin-up and spin-down particles-which are labeled by the indices $i_{\uparrow}=1, \ldots, N_{\uparrow}$ and $i_{\downarrow}=N_{\uparrow}+1, \ldots, N$, respectively, and $N=N_{\uparrow}+N_{\downarrow}$ is the total atom number. The one-dimensional coupling constant 
$g=-2 \hbar^{2} /\left(m a_{1 D}\right)$ is related to the one-dimensional scattering length $a_{1 D}$. We consider repulsive interactions $g \geqslant 0$. In experiments realized in tightly confining cigar-shaped waveguides, sufficiently strong to enter the regime where the gas is kinematically one dimensional, the coupling constant $g$ can be related to the experimental parameters [33], such as the three-dimensional $s$-wave scattering length and the radial harmonic confining frequency. It is convenient to introduce the interaction parameter $\gamma=m g /\left(\hbar^{2} n\right)=2 /\left(n\left|a_{1 D}\right|\right)$, where $n=N / L$ is the density. The external potential $v(x)=$ $V\left[\sin ^{2}\left(\pi x / d_{s}\right)+\sin ^{2}\left(\pi x / d_{l}\right)\right]$ is the superposition of two optical lattices, one with the (short) period $d_{s}$, the other with the (long) period $d_{l}$.

To form an infinite quasiperiodic potential; that is, a deterministic but aperiodic modulation, one should set the ratio of the two periods to be an irrational (Diophantine [34]) number [35]. However, in a finite-size continuous system such choice is incompatible with the use of periodic boundary conditions, which are in fact adopted in our calculations. The best remedy consists in choosing ratios of pairs of coprime integer numbers which, in the thermodynamic limit, converge to an irrational number. One convenient choice [35,36] is to set $r=d_{l} / d_{s}=K_{k+1} / K_{k}$, where the integer sequence $\left\{K_{k}\right\}$ (with $k=0,1, \ldots)$ is the Fibonacci sequence (defined by the rule $K_{k+2}=K_{k+1}+K_{k}$, with $K_{0}=1$ and $K_{1}=1$ ), in which case the limiting value for $k \rightarrow \infty$ is the golden ratio: $r \rightarrow \phi \cong$ 1.618 03; if the system size is fixed as $L=K_{k+1} d_{s}=K_{k} d_{l}$, as we do in our calculations, the potential $v(x)$ complies with periodic boundary conditions, still being aperiodic within the finite box of length $L$. The intensity of the two optical lattices $V$ plays the role of quasidisorder strength. Notice that also other coprime ratios $K_{k+1} / K_{k}$, not taken from the standard Fibonacci sequence, can give similar values of period ratio $r \simeq \phi$, and will be considered in our analysis.

Before addressing the (interacting) many-fermion system, we inspect the properties of the single-particle eigenstates $\psi_{j}(x)$ of the quasiperiodic potential $v(x)$ (which we label with the index $j=1,2, \ldots$ for increasing eigenenergies). We compute them by performing exact diagonalization of the finite Hamiltonian matrix obtained by introducing a fine discretization in the continuous space [37]. To quantify the spatial extent of the single-particle eigenstates, we compute the normalized participation ratio $P=1 / \int_{0}^{L} d x\left|\psi_{j}(x)\right|^{4}$. Ergodic extended states are characterized by large values of the participation ratio, diverging in the thermodynamic limit as $P \propto L$ (in one dimension); instead, for localized states, $P$ is essentially independent of $L$, for sufficiently large systems [38]. In Fig. 1 we display the $P$ value as a function of the eigenstate index $j$ and of the disorder strength $V$. A sudden drop is noticeable around $V \approx 1.2 E_{r s}$ [where $E_{r s}=$ $\pi^{2} \hbar^{2} /\left(2 m d_{s}^{2}\right)$ is the recoil energy of the short-period lattice, which is chosen to be the energy unit, while $d_{s}$ is used as the length unit], slightly depending on $j$, signaling an Anderson localization transition where the single-particle eigenstates become spatially localized. Furthermore, for $V \gtrsim 1.2 E_{r s}$, a sudden increase of $P$ for $j>M_{l}\left(M_{l}=L / d_{l}\right.$ in the number of periods of the long-period optical lattice; similarly $\left.M_{s}=L / d_{s}\right)$ is clearly visible, indicating a mobility edge separating the localized states with $j \leqslant M_{l}$, from extended states with $j>M_{l}$. This feature distinguishes the continuous-

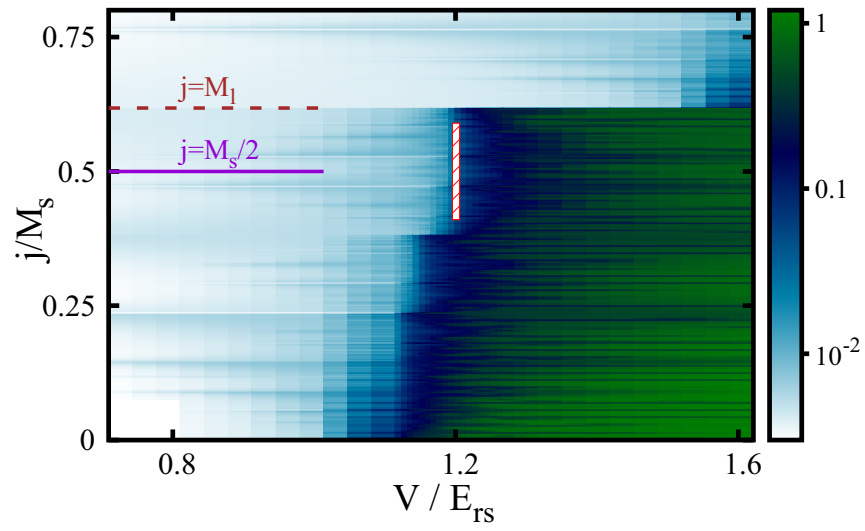

FIG. 1. Logarithmic color-scale plot of the inverse participation ratio of the single-particle eigenstates $d_{s} / P$ as a function of the rescaled eigenstate index $j / M_{s}$ and of the quasidisorder intensity $V / E_{r s}$, i.e., the intensity of the two optical lattices. $M_{s}=L / d_{s}=610$ and $M_{l}=M_{s} / r=377$ are the number of periods of the short-period and of the long-period lattices, respectively; $E_{r s}$ is the recoil energy corresponding to the former. The ratio of the two optical-lattice periods is $d_{l} / d_{s}=r \cong 1.61803$, i.e., close to the golden ratio. The continuous horizontal (violet) segment indicates the index of the highest-occupied orbital for a density so that the short-period lattice is half filled, while the dashed (brown) horizontal segment the one so that the long-period lattice is fully filled. The vertical (red) bar with diagonal pattern indicates the Anderson localization transition of the states with index $j \simeq M_{s} / 2$.

space model we consider from the related Aubry-André model (i.e., a tight-binding discrete-lattice model with an incommensurate potential), where there are no mobility edges, meaning that the whole spectrum localizes at the critical quasidisorder strength [29,30]. In fact, it has previously been found that extended Aubry-André models which include beyond-nearest-neighbor hopping processes, as well as other continuous-space quasiperiodic models similar to ours, host mobility edges [36,39]. Below we consider a spin-populationbalanced (i.e., with $N_{\uparrow}=N_{\downarrow}=N / 2$ ) many-fermion system with density $n=1 / d_{s}$, meaning that the short-period lattice is half filled $\left(N=M_{s}\right)$. At this density, the highest-occupied orbital-whose energy corresponds to the Fermi energyhas the index $j=M_{s} / 2$. To precisely pinpoint the critical quasidisorder strength where the Anderson localization occurs at this energy, we perform a finite-size scaling analysis of the $P$ values; see Fig. 2 . In the inset, the scaling behavior for two values of the quasidisorder strength are shown. To reduce fluctuations due to finite-size effects, we average $P$ values for $M_{s} / 40$ states with index around $j=M_{s} / 2$. The scaling behavior is opposite, saturating to a finite value for the larger $V$, diverging with system size for the smaller $V$. This allows us to locate the critical point $V_{c}^{0}$ of the Anderson (metal-insulator) transition in the narrow interval between the two $V$ values: $1.1956 E_{r s}<V_{c}^{0}<1.2057 E_{r s}$. It is worth mentioning that the Anderson localization transition would occur also for different (irrational) values of the ratio $r$, and that it is visible (in the form of a very sharp crossover) also in large finite-size systems as long as $r$ is not a simple rational number [35]. However, the critical quasidisorder strength where it occurs smoothly varies with $r$ [30]. 


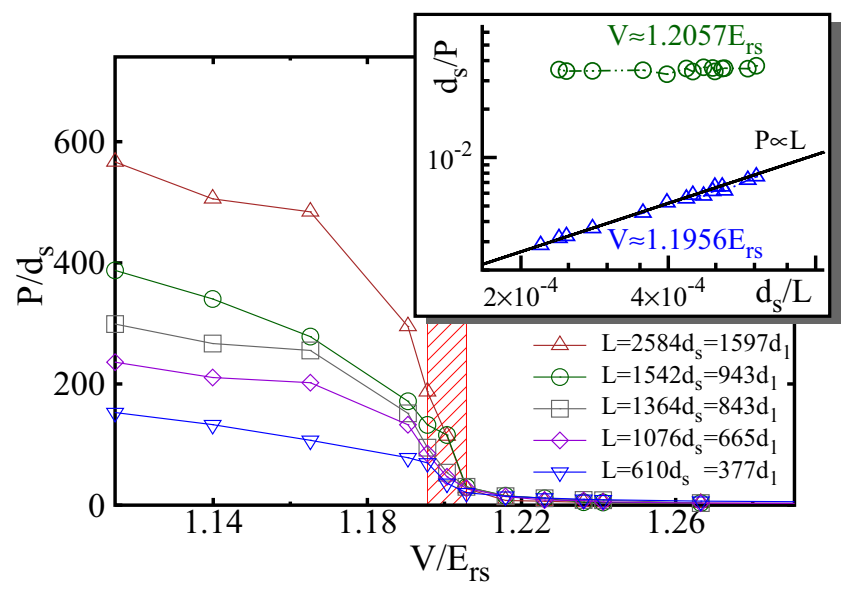

FIG. 2. The main panel shows the participation ratio $P$ of the single-particle eigenstate labeled $j=M_{s} / 2$ as a function of the quasidisorder strength $V / E_{r s}$, for different system sizes $L . d_{s}$ and $d_{s}$ are the period lengths of the short-period and of the long-period lattices, respectively. The vertical (red) bar with diagonal pattern indicates the location of the Anderson transition. The inset shows the scaling of the inverse participation ratio $d_{s} / P$ as a function of the inverse system size $d_{s} / L$, for two values of the quasidisorder strength: in one case $P$ saturates for large system sizes, whereas in the other case it diverges as $P \propto L$ (see continuous black line). These two values bracket the critical point, and they determine the width of the (red) bar in the main panel.

While the single-particle analysis reported above is suitable to identify the insulator transition in noninteracting disordered systems, for the interacting case we need a different approach. We tackle this problem by adopting the tools from the modern theory of the insulating state (see Ref. [40] for a review focusing on its geometrical concepts). This theory was initiated by Kohn's 1963 seminal article [31], where he first proposed that insulation results from the organization of the electrons in the many-particle ground state and that insulators can be identified without inspecting excited-state properties (as in the conventional theory of band insulators), nor the spatial extent of the single-particle eigenstate at the Fermi energy (as in the theory of noninteracting Anderson insulators). Resta and Sorella [32], and later Souza, Wilkens, and Martin [41], developed a rigorous formalism, which has already proven successful to identify band, Mott [32], as well as Anderson insulators [42], both in the case of uncorrelated random disorder and also in systems with correlated disorder, with quasiperiodic potentials, and in quasicrystals [43,44]. This formalism is suitable for $a b$ initio computational techniques such as quantum Monte Carlo simulations [45,46].

It has emerged that, to discern insulators from metals, one has to compute the expectation value $Z=\langle\Psi|\hat{U}| \Psi\rangle(|\Psi\rangle$ is the many-body ground state) of the many-body phase operator $\hat{U}=\exp [i(2 \pi / L) \hat{X}]$, where $\hat{X}=\sum_{i=1}^{N} x_{i} \cdot|Z|$ is the figure of merit to distinguish the two phases. The theory predicts that $|Z| \rightarrow 0$ in the thermodynamic limit $(L \rightarrow \infty$ at fixed $n$ ) for metals, while $|Z| \rightarrow 1$ for insulators. Furthermore, one can define a many-particle localization length $\lambda$ as $\lambda^{2}=$ $-\frac{L^{2}}{4 \pi^{2} N} \ln \left(|Z|^{2}\right)$. For metals, $\lambda$ diverges in the thermodynamic
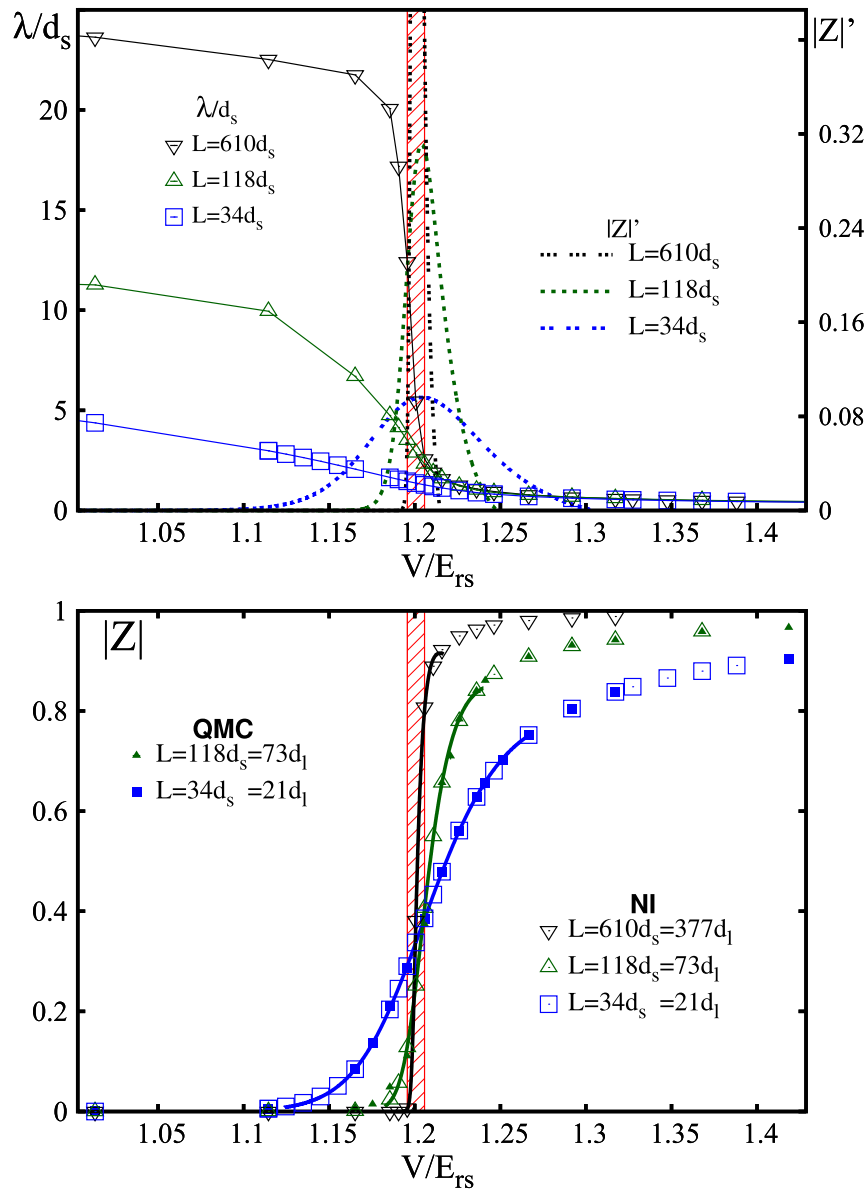

FIG. 3. The lower panel shows the modulus of the expectation value of the many-body phase operator $|Z|$ as a function of the quasidisorder strength $V / E_{r s}$, for different system sizes $L$. Full and empty symbols correspond to QMC and numerical-integration (NI) data, respectively. The continuous curves are the empirical fitting functions (see text). The vertical (red) bar indicates the Anderson transition. The density $n=1 / d_{s}$ is fixed so that the short-period lattice is half filled, the period-lengths ratio is $r \simeq 1.618$. The upper panel shows the empty symbols with connecting lines indicate the many-particle localization length $\lambda / d_{s}$ (left vertical axis), while the dashed curves indicate the (rescaled) derivative of $|Z|$ with respect to $V / E_{r s}$ (right vertical axis).

limit, while it saturates to a finite value for insulators for sufficiently large systems.

In a noninteracting many-fermion system, the ground-state many-body wave function is the Slater determinant $D(N)$ of the first $N$ single-particle spin-orbitals, which involve the first $N / 2$ single-particle spatial wave functions $\psi_{i}(x)$. In this case, the expectation value $Z$ is readily evaluated as $Z=(\operatorname{det} S)^{2}$ where $S$ is the $N / 2 \times N / 2$ matrix of the overlaps $S_{i j}=\int d x \psi_{j}^{*}(x) \psi_{i}(x) \exp (i 2 \pi x / L)$. Alternatively, $Z$ can be computed via a QMC simulation that samples the modulus squared of the exact wave function $\Psi_{\mathrm{NI}}(X)=D\left(N_{\uparrow}\right) D\left(N_{\downarrow}\right)$ $\left[X=\left(x_{1}, \ldots, x_{N}\right)\right.$ is the spatial configuration], where the Slater determinants of the spin-up and spin-down components are separately written for computational efficiency. In Fig. 3 we show data for $|Z|$ and $\lambda$ obtained with both techniques (which we refer to as numerical integration and QMC 
simulation, respectively) as a function of the quasidisorder strength $V$ for different system sizes $L$. These results confirm the expectations; in particular, $|Z|$ decreases with $L$ for small $V$, while it increases saturating to $|Z|=1$ for strong quasidisorder. To pinpoint the metal-insulator transition by using the finite- $L$ data, where the $|Z|$ vs $V$ curve is smooth-as opposed to the thermodynamic limit, in which case a sudden jump develops-we consider two criteria: The first consists of identifying the critical point with the location of the crossing of dataset corresponding to different system sizes. The second consists of identifying the critical point with the location of the maximum of the derivative of the curve $|Z|(V)$ with respect to $V$ (indicated as $|Z|^{\prime}$ ) which, for sufficiently large $L$, would accurately approximate the position where the derivative diverges in the thermodynamic limit. To locate this point, we fit the data with an empirical fitting function based on a modified hyperbolic tangent function:

$$
|Z|(V)=\frac{\exp \left[a_{1}(V-c)\right]-\exp \left[-b_{1}(V-c)\right]}{\exp \left[a_{2}(V-c)\right]-\exp \left[-b_{2}(V-c)\right]},
$$

where the $a_{i}, b_{i}(i=1,2)$, and $c$ are fitting parameters. As is evident from Fig. 3, both criteria provide accurate estimates of the critical quasidisorder strength, in excellent agreement with the predictions based on the system-size scaling of the $P$ values discussed above, even for the relatively small system sizes amenable to the QMC simulations.

To determine $|Z|$ for the interacting many-fermion system, we employ QMC simulations based on the diffusion Monte Carlo algorithm [47]. This projective technique stochastically solves the imaginary-time Schrödinger equation and allows one to sample the exact ground-state wave function. To circumvent the sign problem, which would hinder manyfermion simulations, one has to introduce the fixed-node constraint, meaning that the ground-state wave function is forced to have the same nodes as those of a trial wave function. While in generic higher-dimensional systems this constraint would possibly introduce an uncontrolled approximation, in the one-dimensional case this approach is, in fact, exact (as was shown in Ref. [48]), because (only in one dimension) the nodal surface is equivalent to two identical fermions occupying the same point; this implies that the wave function $\Psi_{\mathrm{NI}}(X)$ defined above has the same nodes as the exact ground state [49,50]. To compute the unbiased expectation value of $\hat{U}$ we employ the standard forward-walking technique [51]. Therefore, the data reported in this paper are free of systematic approximations. To reduce the stochastic fluctuations, we employ the importance sampling technique with the trial wave function written in the Jastrow-Slater form: $\Psi_{T}(X)=\Psi_{\mathrm{NI}}(X) \prod_{i_{\uparrow} i_{\downarrow}} f\left(\mid x_{i_{\uparrow}}-\right.$ $\left.x_{i_{\downarrow}} \mid\right)$ which, beyond the Slater-determinant part $\Psi_{\mathrm{NI}}(X)$ that fixes the nodes, includes a Jastrow correlation function $f(x)>$ 0 that has to ensure the Bethe-Peierls boundary condition $\partial \Psi /\left.\partial\left(x_{i_{\uparrow}}-x_{i_{\downarrow}}\right)\right|_{0}=-\Psi / a_{1 D}$ but is arbitrary otherwise (the specific choice affects only the statistical fluctuations).

In the diffusion Monte Carlo simulations, we consider the system sizes $L=38 d_{s}=23 d_{l}, L=58 d_{s}=35 d_{l}$, and for the weakest interactions also $L=78 d_{s}=47 d_{l} . r=d_{l} / d_{s}$ is again the ratio of two coprime integers, but with the value $r \cong 1.65$, which is slightly larger than the golden ratio $r \cong \phi$ considered above. This value is chosen for numerical convenience, since it allows us to exploit the largest system sizes amenable to the

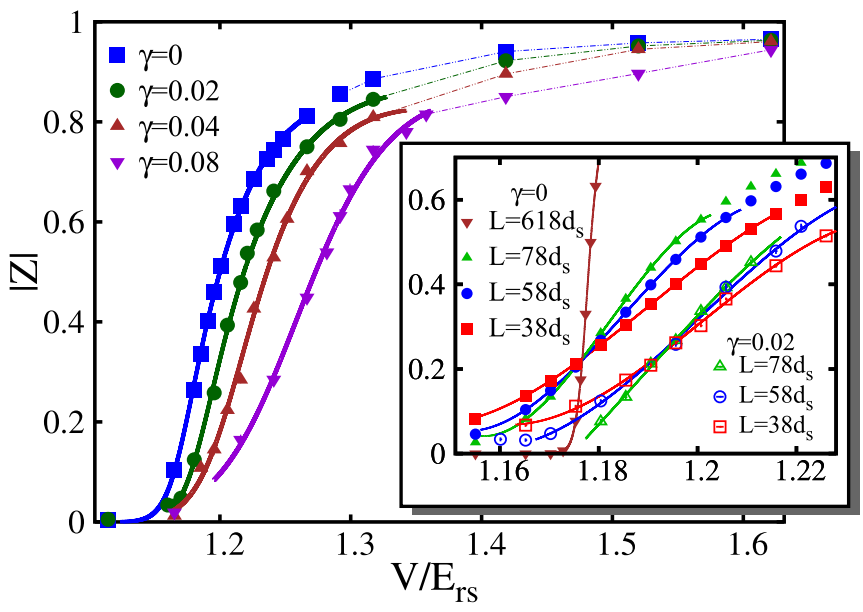

FIG. 4. The main panel shows the modulus of the expectation value of the many-body phase operator $|Z|$ as a function of the quasidisorder strength $V / E_{r s}$, for the same system size $L=58 d_{s}$ but different interaction strengths $\gamma$. The density $n=1 / d_{s}$ is fixed so that the short-period lattice is half filled, the period-length ratio is $r \simeq 1.65$. The full line shows modified hyperbolic tangent (see text). The inset shows the finite-size scaling analysis of $|Z|$ for the noninteracting case $\gamma=0$ (full symbols) and for an interacting case with $\gamma=0.02$ (empty symbols). Continuous curves are cubic fitting functions shown to guide the eye.

QMC simulations. In the noninteracting case (for which we use the numerical integration approach) we also consider the size $L=618 d_{s}=373 d_{l}$. Larger systems cannot be addressed via QMC simulations due to the glassy nature of the insulating phase, which causes a pathological slowdown of the QMC dynamics. It is possible that this problem could be alleviated by using a grand-canonical QMC scheme or by introducing cluster Monte Carlo updates. In Fig. 4 we show the results for $|Z|$ as a function of $V$, obtained for different values of the interaction strength $\gamma$ for the same system size. As $\gamma$ increases, the datasets are shifted towards significantly stronger quasidisorder, giving a qualitative indication that interactions favors metallic behavior. To quantify this effect, we determine the critical quasidisorder strength $V_{c}$ which separates the metal from the insulator by using the two criteria (crossings and peaks of derivative) described above in the noninteracting case. The inset of Fig. 4 displays the finite-size scaling analysis for the interaction parameter $\gamma=0.02$, compared with the noninteracting case $\gamma=0$. In the interacting case, the crossing of the curves-which we identify with the critical quasidisorder, according to the first criterion-is clearly drifted towards larger values of $V$ compared with the noninteracting case (notice that for $r \simeq 1.65$ the metal-insulator transition occurs at slightly weaker quasidisorder than in the case $r \simeq \phi$ considered before), confirming that even interactions as weak as $\gamma=0.02$ determine a positive shift of $V_{c}$. This is the main result of this work.

The zero-temperature phase diagram as a function of quasidisorder strength $V$ and interaction parameters $\gamma$, including the metallic and the insulating phases, is displayed in Fig. 5; the critical quasidisorder strengths determined by using the two criteria are compared, finding precise agreement. These data turn out to be well described by a simple linear fitting 


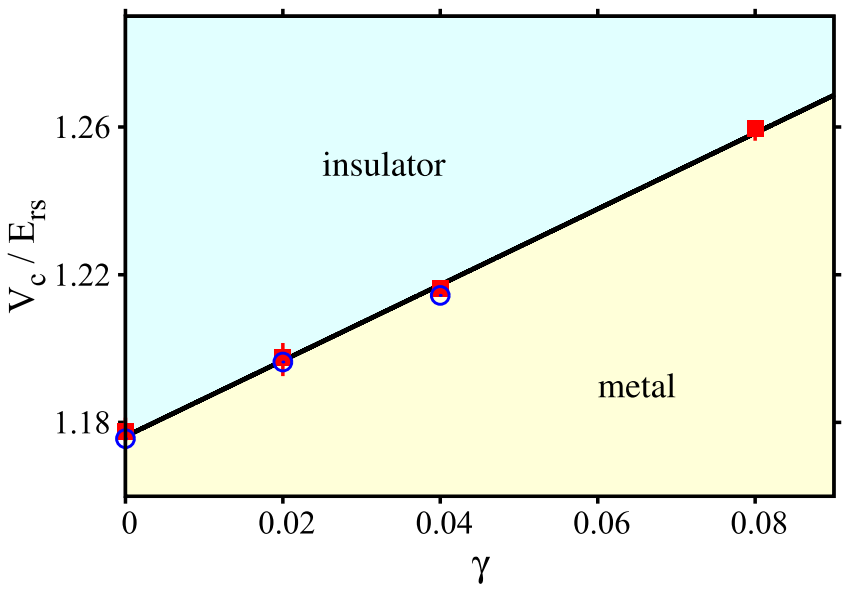

FIG. 5. Critical quasidisorder strength $V_{c} / E_{r s}$, which separates the metallic phase (yellow region) from the insulating phase (cyan region), as a function of the interaction strength $\gamma$. The density is $n=1 / d_{s}$, the optical lattice period is $r \simeq 1.65$. The empty (blue) circles indicate the data obtained from the crossing of the $|Z|(V)$ curves for different system sizes; the full (red) squares are those obtained from the maximum of the derivative of these curves. The continuous (black) line $V_{c} / E_{r s}=1.03(3) \gamma+1.176(2)$ is a linear fit to the latter dataset.

function, suggesting that even infinitesimal interactions affect the location of the metal-insulator transition. These results could serve as a benchmark for experiments performed in shallow bichromatic optical lattices, tuning the interaction parameter via Feshbach resonances. It is worth mentioning that a linear increase of the critical quasidisorder for weak repulsion was previously obtained for the Aubry-André model within the self-consistent Hartree-Fock approximation [43]. It is likely that this linear increase would cease to hold for strong interactions $\gamma \gtrsim 1$; this regime is, however, beyond the scope of this work. An upward shift of the critical point at weak interaction was found also in bosonic systems (see, e.g., Refs. $[19,52])$, in apparent contrast with the argument of Ref. [14] which predicts the opposite behavior in bosons and fermions. Previous results for spinless fermions [53] cannot be directly compared with the (experimentally relevant) spinful fermion case addressed in this work, because the two cases are expected to display important differences $[54,55]$.

In conclusion, we have investigated the effect of weak repulsive contact interaction on the Anderson localization transition in a one-dimensional atomic spinful Fermi gas exposed to a quasiperiodic potential. Our results clearly indicate that even weak repulsions induce a (seemingly linear) drift of the metal-insulator transition towards stronger quasidisorder. This study parallels previous investigations of ultracold atoms in shallow optical lattices [56-62], which explored regimes where simple (nearest-neighbor) tight-binding approximations are not applicable $[39,63]$. The computational approach we implemented, which is based on the modern theory of the insulating state combined with projective QMC simulations, is a promising tool to further explore zero-temperature localization phenomena in complex experimental configurations; in particular, in the relatively unexplored higher-dimensional and continuous-space settings [64]. One can also envision extending the use of the modern theory of the insulating state beyond the ground state in combination with different computational techniques.

We thank G. E. Astrakharchik, R. Fazio, M. Holzmann, V. E. Kravtsov, and U. Schneider for useful discussions. S.P. acknowledges financial support from the EU-H2020 Project No. 641122 QUIC - Quantum simulations of insulators and conductors.
[1] P. W. Anderson, Phys. Rev. 109, 1492 (1958).

[2] E. Abrahams, P. W. Anderson, D. C. Licciardello, and T. V. Ramakrishnan, Phys. Rev. Lett. 42, 673 (1979).

[3] J. Billy, V. Josse, Z. Zuo, A. Bernard, B. Hambrecht, P. Lugan, D. Clément, L. Sanchez-Palencia, P. Bouyer, and A. Aspect, Nature (London) 453, 891 (2008).

[4] G. Roati, C. D'Errico, L. Fallani, M. Fattori, C. Fort, M. Zaccanti, G. Modugno, M. Modugno, and M. Inguscio, Nature (London) 453, 895 (2008).

[5] F. Jendrzejewski, A. Bernard, K. Mueller, P. Cheinet, V. Josse, M. Piraud, L. Pezzé, L. Sanchez-Palencia, A. Aspect, and P. Bouyer, Nat. Phys. 8, 398 (2012).

[6] S. S. Kondov, W. R. McGehee, J. J. Zirbel, and B. DeMarco, Science 334, 66 (2011).

[7] W. R. McGehee, S. S. Kondov, W. Xu, J. J. Zirbel, and B. DeMarco, Phys. Rev. Lett. 111, 145303 (2013).

[8] D. Delande and G. Orso, Phys. Rev. Lett. 113, 060601 (2014).

[9] E. Fratini and S. Pilati, Phys. Rev. A 91, 061601 (2015).

[10] E. Fratini and S. Pilati, Phys. Rev. A 92, 063621 (2015).

[11] M. Pasek, G. Orso, and D. Delande, arXiv:1609.01065.
[12] G. Semeghini, M. Landini, P. Castilho, S. Roy, G. Spagnolli, A. Trenkwalder, M. Fattori, M. Inguscio, and G. Modugno, Nat. Phys. 11, 554 (2015).

[13] A. Aspect and M. Inguscio, Phys. Today 62, 30 (2009).

[14] L. Sanchez-Palencia and M. Lewenstein, Nat. Phys. 6, 87 (2010).

[15] B. Deissler, M. Zaccanti, G. Roati, C. D’Errico, M. Fattori, M. Modugno, G. Modugno, and M. Inguscio, Nat. Phys. 6, 354 (2010).

[16] D. Clément, A. F. Varòn, J. A. Retter, L. Sanchez-Palencia, A. Aspect, and P. Bouyer, New J. Phys. 8, 165 (2006).

[17] L. Tanzi, E. Lucioni, S. Chaudhuri, L. Gori, A. Kumar, C. D'Errico, M. Inguscio, and G. Modugno, Phys. Rev. Lett. 111, 115301 (2013).

[18] S. Krinner, D. Stadler, J. Meineke, J.-P. Brantut, and T. Esslinger, Phys. Rev. Lett. 115, 045302 (2015).

[19] C. D’Errico, E. Lucioni, L. Tanzi, L. Gori, G. Roux, I. P. McCulloch, T. Giamarchi, M. Inguscio, and G. Modugno, Phys. Rev. Lett. 113, 095301 (2014).

[20] S. S. Kondov, W. R. McGehee, W. Xu, and B. DeMarco, Phys. Rev. Lett. 114, 083002 (2015). 
[21] M. Schreiber, S. S. Hodgman, P. Bordia, H. P. Lüschen, M. H. Fischer, R. Vosk, E. Altman, U. Schneider, and I. Bloch, Science 349, 842 (2015).

[22] J.-y. Choi, S. Hild, J. Zeiher, P. Schauß, A. Rubio-Abadal, T. Yefsah, V. Khemani, D. A. Huse, I. Bloch, and C. Gross, Science 352, 1547 (2016).

[23] D. M. Basko, I. L. Aleiner, and B. L. Altshuler, Ann. Phys. (NY) 321, 1126 (2006).

[24] The notion of temperature in the many-body localized phase is ambiguous since a statistical mechanics ensemble is believed to be inapplicable.

[25] V. Oganesyan and D. A. Huse, Phys. Rev. B 75, 155111 (2007).

[26] V. P. Michal, I. L. Aleiner, B. L. Altshuler, and G. V. Shlyapnikov, Proc. Natl. Acad. Sci. USA 113, E4455 (2016).

[27] T. Giamarchi and H. J. Schulz, Phys. Rev. B 37, 325 (1988).

[28] S. Aubry and G. André, Ann. Isr. Phys. Soc. 3, 18 (1980).

[29] D. J. Boers, B. Goedeke, D. Hinrichs, and M. Holthaus, Phys. Rev. A 75, 063404 (2007).

[30] J. Biddle, B. Wang, D. J. Priour Jr., and S. Das Sarma, Phys. Rev. A 80, 021603(R) (2009).

[31] W. Kohn, Phys. Rev. 133, A171 (1963).

[32] R. Resta and S. Sorella, Phys. Rev. Lett. 82, 370 (1999).

[33] M. Olshanii, Phys. Rev. Lett. 81, 938 (1998).

[34] S. Y. Jitomirskaya, Ann. Math. 150, 1159 (1999).

[35] M. Modugno, New J. Phys. 11, 033023 (2009).

[36] R. B. Diener, G. A. Georgakis, J. Zhong, M. Raizen, and Q. Niu, Phys. Rev. A 64, 033416 (2001).

[37] The second derivative is approximated by using an 11-point formula and 20-30 points per $d_{s}$.

[38] B. Kramer and A. MacKinnon, Rep. Prog. Phys. 56, 1469 (1993).

[39] J. Biddle, D. J. Priour, Jr., B. Wang, and S. Das Sarma, Phys. Rev. B 83, 075105 (2011).

[40] R. Resta, Eur. Phys. J. B 79, 121 (2011).

[41] I. Souza, T. Wilkens, and R. M. Martin, Phys. Rev. B 62, 1666 (2000).

[42] G. L. Bendazzoli, S. Evangelisti, A. Monari, and R. Resta, J. Chem. Phys. 133, 064703 (2010).

[43] V. Kerala Varma and S. Pilati, Phys. Rev. B 92, 134207 (2015).

[44] V. Kerala Varma, S. Pilati, and V. E. Kravtsov, Phys. Rev. B 94, 214204 (2016).
[45] L. Stella, C. Attaccalite, S. Sorella, and A. Rubio, Phys. Rev. B 84, 245117 (2011).

[46] N. Hine and W. Foulkes, J. Phys.: Condens. Matter 19, 506212 (2007).

[47] P. J. Reynolds, D. M. Ceperley, B. J. Alder, and W. A. Lester Jr, J. Chem. Phys. 77, 5593 (1982).

[48] D. M. Ceperley, J. Stat. Phys. 63, 1237 (1991).

[49] M. Casula, D. M. Ceperley, and E. J. Mueller, Phys. Rev. A 78, 033607 (2008).

[50] N. Matveeva and G. Astrakharchik, New J. Phys. 18, 065009 (2016).

[51] J. Boronat, in Microscopic Approaches to Quantum Liquids in Confined Geometries, edited by E. Krotscheck and J. Navarro (World Scientific, Singapore, 2002), Chap. 2, p. 21.

[52] S. Lellouch and L. Sanchez-Palencia, Phys. Rev. A 90, 061602 (2014).

[53] P. Naldesi, E. Ercolessi, and T. Roscilde, SciPost Phys. 1(1), 010 (2016).

[54] P. Prelovšek, O. Barišić, and M. Žnidarič, Phys. Rev. B 94, 241104 (2016).

[55] V. Mastropietro, Annalen der Physik (2016), doi: 10.1002/andp.201600270.

[56] E. Haller, R. Hart, M. J. Mark, J. G. Danzl, L. Reichsöllner, M. Gustavsson, M. Dalmonte, G. Pupillo, and H.-C. Nägerl, Nature (London) 466, 597 (2010).

[57] S. Pilati and M. Troyer, Phys. Rev. Lett. 108, 155301 (2012).

[58] F. De Soto and M. C. Gordillo, Phys. Rev. A 85, 013607 (2012).

[59] G. E. Astrakharchik, K. V. Krutitsky, M. Lewenstein, and F. Mazzanti, Phys. Rev. A 93, 021605 (2016).

[60] G. Boéris, L. Gori, M. D. Hoogerland, A. Kumar, E. Lucioni, L. Tanzi, M. Inguscio, T. Giamarchi, C. D’Errico, G. Carleo et al., Phys. Rev. A 93, 011601 (2016).

[61] M. C. Gordillo, C. Carbonell-Coronado, and F. De Soto, Phys. Rev. A 91, 043618 (2015).

[62] S. Pilati, I. Zintchenko, and M. Troyer, Phys. Rev. Lett. 112, 015301 (2014).

[63] V. Kerala Varma and R. J. Sánchez, Phys. Rev. A 92, 013618 (2015).

[64] I. Gornyi, A. Mirlin, M. Müller, and D. Polyakov, arXiv:1611.05895. 